Mirjam Aeschbach*

\title{
Politisierung von Kultur, Religion, und Geschlecht: Die Kulturalisierungen eines verweigerten Handschlags in Deutschschweizer Medien
}

https://doi.org/10.1515/zfr-2019-0015

Zusammenfassung: In der Deutschschweizer massenmedialen Debatte zur Weigerung zweier Schüler in der Gemeinde Therwil, ihrer Lehrerin die Hand zu schütteln, wird häufig der Kulturbegriff verwendet. Der vorliegende Beitrag analysiert, wie „Kultur“ als wissensstrukturierende Kategorie in der Konstituierung der Gruppenzugehörigkeiten zur Schweizer Mehrheitsgesellschaft und zur muslimischen Minderheit zum Tragen gekommen ist. Basierend auf dem Konzept der „Kulturalisierung“ untersucht die qualitative Medienanalyse die verschiedenen „Spielarten der Kulturalisierung“ (Teczan 2011), die in der Debatte um den Fall Therwil verwendet wurden. Die Ergebnisse zeigen, dass „Kultur“ in Beziehung zu geografischer Herkunft, religiöser Zugehörigkeit und spezifischen Wertvorstellungen gesetzt wurde. Gleichzeitig kann die Verknüpfung dieser Ideen mit Vorstellungen „kulturspezifischer“ Geschlechterverhältnisse und angenommenen Einstellungen zur Geschlechtergerechtigkeit und sexuellen Liberalisierung beobachtet werden.

Schlagwörter: Kulturalisierung, Religion und Medien, Geschlecht, Islam, Schweiz

Abstract: In the Swiss-German media debate on the refusal of two male students in the municipality of Therwil to shake the hand of their female teacher, the term culture is often referred to. The present contribution examines how "culture", as a knowledge-structuring category, figures in the constitution of group affiliations to the Swiss majority society and to the Muslim minority. Based on the concept of "culturalization", the qualitative media analysis examines the various "versions of culturalization" (Teczan 2011) used in the debate surrounding the Therwil case. The results of this analysis show that "culture" was constructed in relation to ideas of geographical origin, religious affiliation, and specific values. At the same

\footnotetext{
*Kontaktperson: Mirjam Aeschbach, Religionswissenschaftliches Seminar Universität Zürich, Kantonsschulstrasse 1, 8001 Zürich, E-Mail: mirjam.aeschbach@rws.uzh.ch
} 
time, these ideas were linked to notions of "culture-specific" gender relations and assumed attitudes towards gender equality and sexual liberalization.

Keywords: Culturalization, Religion and Media, Gender, Islam, Switzerland

\section{Einleitung}

„Der Handschlag ist Teil unserer Kultur [...] so habe ich mir Integration nicht vorgestellt.“ Mit diesen Worten kommentierte die Schweizer Bundesrätin Simonetta Sommaruga im Schweizer Fernsehen die Weigerung zweier Schüler in der Basler Gemeinde Therwil, ihrer Lehrerin die Hand $\mathrm{zu}$ schütteln (SRF, 10 vor 10, 04.04.16). Damit erhob sie den Handschlag zum Schweizer Kulturgut und deutete die Handschlagverweigerung als Problem der Integration. In der anschließenden medialen Berichterstattung wurde diese Deutung des Vorfalles vorwiegend aufgenommen und das Ereignis entwickelte sich zu einem temporären Schwerpunkt in der Debatte über akzeptable Differenzen im Hinblick auf die muslimische Minderheit in der Schweiz. Dabei wurde häufig mit dem Kulturbegriff operiert, um einerseits die muslimische Minderheit und andererseits die Schweiz als Kollektiv $\mathrm{zu}$ fassen und hinsichtlich geographischer Herkunft, Religion, Praktiken und Wertvorstellungen zu deuten. In diesem Artikel wird die Diskussion des TherwilFalles in öffentlichen Deutschschweizer Medieninstitutionen untersucht, ${ }^{1}$ insbesondere in deutschsprachigen Zeitungen und dem Schweizer Radio und Fernsehen (SRF), hinsichtlich der Art und Weise, wie der Kulturbegriff in dieser Berichterstattung als wissensstrukturierende Kategorie verwendet wurde.

Massenmedien als Organe einer Politöffentlichkeit haben den Anspruch, aktuelles, notwendiges Wissen zu generieren und die Funktion der Selbstbeobachtung der Gesellschaft auszuüben (Behloul 2011, 139, Pfetsch und Marcinkowski 2009, 11). Allerdings sind die Publikationen medialer Öffentlichkeiten keine reinen Beobachtungsprozesse, sondern produzieren vielmehr selbst Diskurse ${ }^{2}$, die Realität nicht nur abbilden, sondern auch bilden (Dreesen, Kumięga und Spieß 2012, 11). In dieser Weise können Vorkommnisse mit Bedeutung angereichert zu Brennpunkten medialer Debatten werden (Langenohl 2011, 84). Langenohl erläutert den Umstand, dass solche medialen Kristallisationspunkte nicht als Ereignis-

1 Für eine Analyse der länderübergreifenden medialen Verbreitung des Falles Therwil siehe Stahel in dieser Zeitschriftenausgabe.

2 Diskurs wird hier in seiner foucaultschen Ableitung verstanden (Dreesen, Kumięga und Spieß 2012, Fraas und Klemm 2005), als „Ausdruck wie Konstitutionsbedingung des Sozialen“ (Fraas, Meier und Pentzold 2014, 12). 
se vorliegen, sondern zu solchen in der Medienöffentlichkeit „verfertigt werden“ (2011, 84), mit Verweis auf Foucaults Begriff der Evenementalisierung von Gegebenheiten (Foucault 2009). ${ }^{3}$ Medial verfertigte Ereignisse sind erkennbar in Kommunikationsverdichtungen, in denen als Schlüsselereignisse dargestellte Geschehnisse zeitweilig die Schlagzeilen beherrschen (Schranz und Imhof 2002, 2). In massenmedialer Kommunikation entsteht also ein öffentlicher Raum um den Mittelpunkt verfertigter Ereignisse, in welchem Gruppenzugehörigkeiten und entsprechende Identitätspositionen (re-)produziert und verhandelt werden (Torfing 1999, 210f.). Dabei etablieren sich nicht nur die Identitätsbilder gesellschaftlicher Minderheiten, sondern auch Mehrheitsgesellschaften „offenbaren sich in Form von [öffentlicher] Kommunikation“ (Schranz und Imhof 2002, 1) in massenmedialen Debatten (vgl. Langenohl 2011, 83).

An diese Überlegungen anschließend wird im folgenden Beitrag der Frage nachgegangen, wie der häufig rezipierte Kulturbegriff in der Konstituierung der Gruppenzugehörigkeiten sowohl der Schweizer Mehrheitsgesellschaft als auch der muslimischen Minderheit im Deutschschweizer Mediendiskurs um den Therwil-Fall zum Tragen gekommen ist. Im ersten Teil stelle ich hierfür das Theoriekonzept der „Kulturalisierung“ vor und skizziere Tezcan’s Modell verschiedener „Spielarten der Kulturalisierung“ (2011). In der qualitativen Medienanalyse im zweiten Teil des Beitrages werden diese Spielarten der Kulturalisierung mit Fokus auf die Verstrickungen der Konzepte „Kultur“ und „Geschlecht“ empirisch herausgearbeitet.

\section{Kultur und Kulturalisierung}

Der „chronisch vielfältige Kulturbegriff“ (Reckwitz 2011, 2) wurde seit seiner Entstehung im 18. Jahrhundert in unterschiedlicher Art und Weise in soziopolitischen als auch in wissenschaftlichen Diskursen benutzt (Kleeberg und Langenohl 2011, 281, Reckwitz 2011, 3-8, Tezcan 2011, 357). Die vor allem anfänglich weit verbreitete wissenschaftliche Verwendung von „Kultur“ als totalitätsorientierte Bezeichnung für ,spezifische Lebensformen einzelner Kollektive in der Geschichte“ (Reckwitz 2011, 5) wurde seither von diversen Seiten als kontingenzeinschränkende Tendenz kritisiert, essentialisierende Konstitutionen ganzheitlicher Kollektive zu bilden (Abu-Lughod 1996, Dennerlein 2017, Reckwitz 2011). Reckwitz er-

3 Diese Anlehnung an Foucault findet sich auch bei Ezli, der davon spricht, dass Kultur evenementalisiert, also „zum Ereignis [ge]macht [wird]“ (2010, 9). Im Folgenden werde ich mich auf Langenohls Begrifflichkeit stützen und von „,verfertigten Medienereignissen“ sprechen. 
klärt, dass solch homogenisierende Ansätze, die „Totalitäten menschlicher Lebensweisen in verschiedenen ,Völkern', ,Nationen', ,Gemeinschaften“, ,Kulturkreisen““ $(2011,5)$ mit Verweis auf den Kulturbegriff „sichtbar“ machen wollten, seit dem Perspektivenwechsel zu einem sozialkonstruktivistischen Kulturverständnis im 20. Jahrhundert wissenschaftlich überholt seien (2011, 7-8). ${ }^{4}$ Gleichzeitig weisen jedoch Kleeberg und Langenohl (2011, 284) darauf hin, dass die Entlehnung des Kulturbegriffes in nicht klassisch kulturorientierten Wissenschaftsfeldern die analytische Trennschärfe des Begriffes verwässert habe. Dies könne, so Kleeberg und Langenohl (2011, 282) sowie Tezcan (2011, 357), am Beispiel des Politikwissenschaftlers Samuel Huntington und seinem Werk Kampf der Kulturen ${ }^{5}$ (1996) gesehen werden, welches bis heute zur vermehrten Anwendung des Kulturbegriffes in einer Vielzahl wissenschaftlicher Disziplinen führe. Gleichzeitig ist der Verweis auf „Kultur“ auch in soziopolitischen Bereichen zu beobachten, wo Konflikte zwischen „Mehrheitsgesellschaften und ,ihren“ Minderheiten [...] mit Referenz nicht auf ökonomische oder soziale Ursachen, sondern auf ,Kultur` gedeutet [werden]“ (Kleeberg und Langenohl 2011, 283).

Kritik an einer solch totalitätsorientierten Verwendung des Kulturbegriffes, sowohl im wissenschaftlichen als auch im gesellschaftspolitischen Bereich, wird zunehmend mit dem Konzept der „Kulturalisierung“ geäußert (Kleeberg und Langenohl 2011, Langenohl 2011, Tezcan 2011). Tezcan unterscheidet dabei zwischen vier „Spielarten der Kulturalisierung“: Er definiert „Kulturalisierung“ als „einen angenommenen kausalen Zusammenhang zwischen dem Ursprung einer als kohärent geltenden sozialen Gruppe und dem sozialen Handeln ihrer Mitglieder“ (2011, 358). Auf diese Weise ist Kulturalisierung zunächst (1) die Zuschreibung einer „kulturellen“ Homogenität an eine kollektive Einheit, beziehungsweise eine Essentialisierung einer Gruppe durch den Begriff „Kultur“. Auf einer zweiten Ebene wird Kulturalisierung zu einer (2) politischen Strategie, die durch den Kulturverweis eingegrenzte Kollektive als politische Subjekte konstruiert (Tezcan 2011, 358; 364). Die dritte Spielart ist der (3) Anspruch der „Kultivierung“, ${ }^{6}$ der den „kulturellen Minderheiten“ gegenüber in Integrationsnarrativen geltend gemacht

4 Reckwitz nennt die vier kulturtheoretischen Herangehensweisen; 1) Phänomenologie und Hermeneutik, 2) Strukturalismus und Semiotik, 3) Pragmatismus und 4) die Sprachspielphilosophie, die alle vier „das Rüstzeug für ein bedeutungsorientiertes, sozialkonstruktivistisches Kulturverständnis [liefern]“ (2011, 7). Die heutigen Versionen der Kulturtheorie basieren vor allem auf den Modellen „Kultur als Diskurse“ und „Kultur als Praktiken“ (Reckwitz 2011, 17).

5 Mehr zu der Gegenüberstellung einer ,westlichen Kultur“ und ,der Kultur des Islams“ (Englisch: Clash of Civilizations) bei Huntington siehe Asad (1997).

6 Der Anspruch auf Kultivierung scheint auf eine normativ ausgerichtete Version des Kulturbegriffes zurückzugehen, in der „,Kultur‘ im Sinne einer menschlichen Lebensweise [...] zunächst noch untrennbar verbunden mit einer Bewertung dieser Lebensweise“ ist und ein „universaler Maßstab 
wird (Tezcan 2011, 358). Hier wird das „Eigene“ und das „Fremde“ im Prozess gesellschaftlicher (Selbst-)Beschreibung bestimmt und ein Anspruch auf Anpassung basierend auf einem normativ ausgerichteten Verständnis von Kultiviertheit, das der eigenen „Kultur“ entspricht, geäußert (Tezcan 2011, 358; 370-373). Auf dieser Ebene kann Kulturalisierung als „transnationale Strategie der Verhandlung von Differenz und Anerkennung“ $(2017,38)$ verstanden und untersucht werden. Auf der letzten Ebene (4) wird „auf die Beschreibung [lokaler] Konflikte als globaler Kulturkonflikt mit einem Kulturalisierungsvorwurf reagiert“ (Tezcan 2011, 358, Hervorhebung der Autorin). Dies wird am Beispiel der Islampolitik in Deutschland deutlich, der die „Kulturalisierung“ von „eigentlich“ sozialen Problemen vorgeworfen wird (Tezcan 2011, 373).

Die Verwendung der ersten drei Spielarten der Kulturalisierung in gesellschaftspolitischen Kontexten wird besonders deutlich im Zusammenhang mit Auseinandersetzungen um die „Leitkultur“ eines Landes und Diskussionen über Integration und Multikulturalismus (Kleeberg und Langenohl 2011, 283). Wissenschaftlerinnen und Wissenschaftler haben hervorgehoben, dass dem Kulturbegriff im Hinblick auf die Konstituierung nationaler Zugehörigkeit und Staatsbürgerschaft im gegenwärtigen europäischen Kontext zunehmende Bedeutung beigemessen wird (El-Tayeb 2011, Kleeberg und Langenohl 2011, Langenohl 2011, Mepschen, Duyvendak und Tonkens 2010). In diesem Zusammenhang bezeichnet Langenohl heutige europäische Gesellschaften als „kulturalisierte Mehrheitsgesellschaften“, in denen die Vorstellung, wer zum gesellschaftlichen Mainstream gehört und wer nicht, sich in einem kulturalistischen Idiom manifestiert (2011, 83). ${ }^{7}$

Studien zu europäischen Gesellschaften haben zudem gezeigt, dass die Art der Bürgerschaft, die konstitutiv für die „kulturalisierte Mehrheitsgesellschaft“ ist, sich im Prozess der Thematisierung von Geschlecht und Sexualität herauskristallisiert (für die Niederlande vgl. Mepschen, Duyvendak und Tonkens 2010, für Frankreich vgl. Fassin 2010, Fassin 2012, für mehrere Kontexte vgl. Butler 2008, El-Tayeb 2011). ${ }^{8}$ Dabei steht das Engagement für den Schutz der Geschlechter-

des ,Kultivierten` angenommen wird, der insgeheim der bürgerlichen Kultur entspricht“ (Reckwitz 2011, 4).

7 Dies stützt Langenohl auf eine Inhaltsanalyse der öffentlichen Debatten zur Minarettinitiative in der Schweiz im November 2009 und zur Publikation des Buchs „Deutschland schafft sich ab“ von Thilo Sarrazin am 30. August 2010 (2011, 84).

8 Als Beispiel für die Instrumentalisierung von Sexualpolitik in Integrationsdebatten zitiert Fassin den französischen Politiker Sarkozy, der Frankreich als eine Wertegemeinschaft bezeichnete, in welcher Frauen genauso „frei“ seien wie Männer (Fassin 2010, 513). Als Beispiel aus dem deutschsprachigen Raum bringt Fassin zudem eine Befragung in Baden-Württemberg an, welche Bewerbende um die Staatsbürgerschaft auf bestimmte „Werte“ prüft, die mit der sexuellen Befreiung der 
gleichstellung und der sexuellen Freiheit im Zentrum der Selbstbeschreibung europäischer Bürgerschaft. Die „Wertefrage, die mit der wachsenden Konjunktur des Kulturellen an politischer Relevanz gewonnen hat“ (Tezcan 2011, 362-363) wird also mit Bezug auf Wertvorstellungen zu Geschlecht und Sexualität abgehandelt. ${ }^{9}$ In diesem Zusammenhang prägte Leticia Sabsay den Begriff „sexuelle Bürgerschaft“, mit dem sie aufzeigt, dass die Einbeziehung von geschlechtsspezifischen und sexuellen „Anderen“ in die euro-amerikanischen Vorstellungen von Staatsbürgerschaft in den letzten Jahrzehnten zur Bildung „of new sexual rightsbearing subjects as if they were already existing entities“ $(2012,608)$ geführt hat. Diese Enthistorisierung der Geschlechtergleichstellung und der sexuellen Liberalisierung sei mit einer spezifischen Vorstellung eines liberalen „Selbst“ verbunden, das sich gegen eine Vielzahl von - oft orientalisierten und durch ihre „Kultur“ homogenisierten - „Anderen“ konstituiert habe und laufend konstituiere (Sabsay 2012). Gleichzeitig werden die „sexual rights-bearing subjects“ häufig in ein Fortschrittnarrativ des „euro-amerikanischen Selbst“ eingebettet, welches sich von den „muslimischen Anderen“, als rückständig und vormodern gekennzeichnet, abgrenzt. Durch die gleichzeitige Kulturalisierung des muslimischen Kollektivs wird dabei, wie Dennerlein aufzeigt, „Geschlechterdifferenz als Kern islamischer Identität [enthistorisiert] und damit gerade auch gegenüber innermuslimischer Kritik [immunisiert]“ $(2017,37)$.

\section{Medienereignis Therwil: Daten und Methode}

Der Datensatz ${ }^{10}$ besteht aus Deutschschweizer Mediendokumenten, die zwischen April 2016 und Dezember 2017 publiziert wurden und sich auf den Therwil-Fall

Gleichstellung der Geschlechter zusammenhängen, um zu bestimmen, ob sie für den Erhalt der Staatsbürgerschaft geeignet sind (2010, 515-516).

9 Nicht nur politisch, sondern auch in wissenschaftlichen Arbeiten sind kulturalisierende Logiken verschränkt mit Geschlecht und Sexualität. Dies ist beispielsweise in der Weiterführung von Huntingtons Clash-of-Civilizations-Paradigma durch Inglehart und Norris (2003) ersichtlich, die mit Verweis auf westliche und muslimische Länder sagen, der wahre „Kampf der Kulturen“ sei der „sexuelle Kampf der Kulturen“. Auch die bereits sehr früh einflussreiche und viel zitierte Frage Okins, „,is multiculturalism bad for women?“ (1999) verweist auf die Grundannahme, dass gewisse „Kulturen“ der „Anderen“ sich hinsichtlich der Wertvorstellungen zu Geschlechtergleichstellung fundamental unterschieden.

10 Die Datenerhebung und -analyse wurde mit Hilfe Altheides Konzeptualisierung der Qualitativen Medienanalyse, auch Ethnographische Inhaltsanalyse (ECA) genannt, durchgeführt (Altheide 1987a, b, Altheide and Schneider 2013). 
beziehen. ${ }^{11}$ Dieser beinhaltet 192 systematisch erhobene Dokumente aus Zeitungen $^{12}$ inklusive Leserkommentaren ${ }^{13}$ und 58 Mediendokumente des SRF. ${ }^{14}$ Den größten Teil der medialen Aufmerksamkeit erhielt der Fall im April 2016, nach seiner ersten medialen Erwähnung, mit 108 Zeitungsdokumenten und 24 Medienoutputs des SRF im entsprechenden Monat (siehe Tabelle 1).

Tabelle 1: Zeitachse Deutschschweizer Medienoutputs zum Therwil-Fall

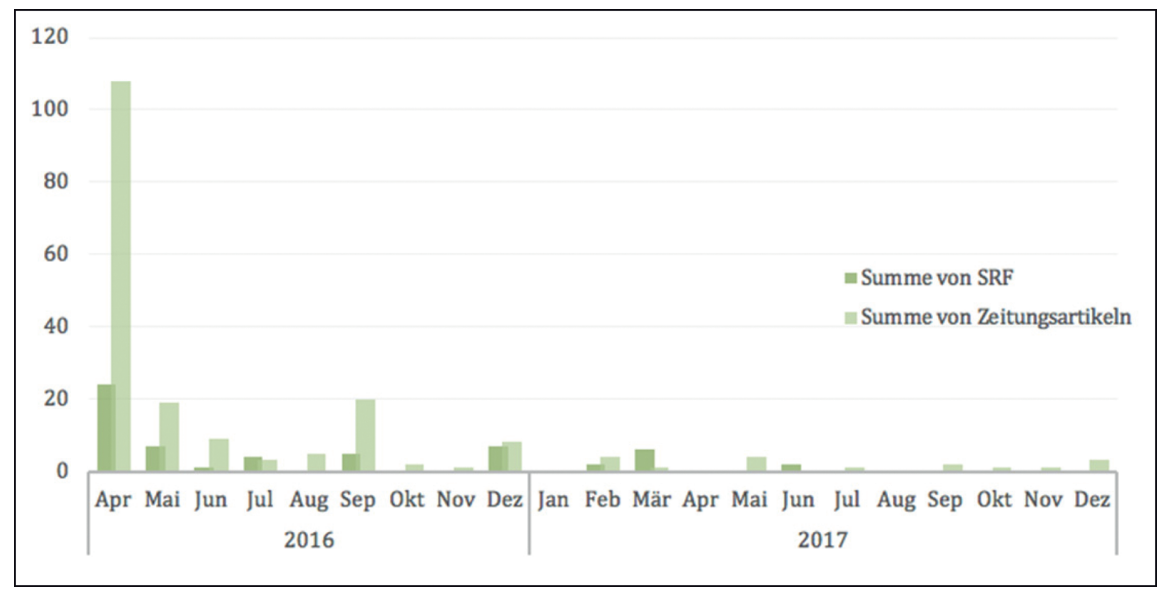

11 Im Sinne Altheides und Schneiders Methode der qualitativen Medienanalyse wurde das Thema das verweigerten Handschlages in der Schulgemeinde Therwil „über einen bestimmten Zeitraum [2016-2017] über verschiedene Nachrichtenmedien hinweg [...] verfolg[t]“ (2012, 117).

12 Die Deutschschweizer Zeitungsartikel wurden einem Gesamtdatensatz aller auf LexisNexis und Factiva eingespeisten, länderübergreifend publizierten Artikel zum verweigerten Handschlag in Therwil entnommen. Mein Dank gilt Lea Stahel, die mir diesen Datensatz zur Verfügung gestellt hat (Stahel 2018).

13 In diesem Beitrag sind in Deutschschweizer Zeitungen publizierte Leserkommentare eingeschlossen, nicht aber Onlinekommentare. In diesem Rahmen publizierte Leserbriefe werden als Beiträge zu öffentlichen Diskursen verstanden (Madeker 2008, 110). Während die Deutungsmacht von Leserkommentaren aufgrund ihres „niedrigeren symbolischen Kapitals“ (Madeker 2008, 118) häufig als schwach eingestuft wird, zeigt beispielsweise die von einem Leserkommentar ausgelöste schweizweite Debatte zu Minaretten, die in der Initiative zum Minarettverbot resultierte, ihren potentiellen Einfluss (Wäckerlig 2014, 18). Von der Wirkungsannahme abgesehen liegt der Schwerpunkt der vorliegenden Arbeit auf der Herausarbeitung der diskursiv geäußerten Deutungszusammenhänge, zu denen Leserbriefe beitragen.

14 Die Dokumente wurden mit Hilfe von Stichwortsuchen zu „Therwil“, „Handschlag“, „Handschlag-Affäre“ und „Händedruck“ dem Online-Archiv des SRF entnommen. 
Die Debatte flachte nach einigen Monaten weitgehend ab, wobei sich die Mediendebatte gegen Ende 2016 und im Jahr 2017 in erster Linie mit der kantonalen Verfassungsänderung befassten. Diese beinhaltete den Satz ,weltanschauliche Auffassungen und religiöse Vorgaben entbinden nicht von der Erfüllung bürgerlicher Pflichten“ und die Verpflichtung der Schulen, „bei wesentlichen Integrationsschwierigkeiten von Schülerinnen und Schülern [...] dem Baselbieter Amt für Migration eine Meldung zu erstatten“ (Vernehmlassung zu Kantonsverfassung und Bildungsgesetz, Baselland, 07.12.16). In diesen Artikeln wurden dabei einige der anfänglichen Rahmungen, die den Fall in häufig kulturessentialistischen Wortlauten als Problem der Integration mit der muslimischen Minderheit in der Schweiz darstellten, kritisch begutachtet und revidiert, was die anfangs gehäufte und sich im Laufe der Debatte verringernde Referenz zum Kulturbegriff erklären könnte (siehe Tabelle 2).

Um die angewandten „Spielarten der Kulturalisierung“ und die Verschränkungen von Geschlecht und Sexualität mit kulturalisierten Selbst- und Fremdbildern innerhalb dieses Medienereignisses herauszuarbeiten, wurde aus dem Gesamtdatensatz der Deutschschweizer Medienoutputs zum Fall Therwil eine theoriegeleitete Auswahl aller Dokumente, welche den Begriff „Kultur“ beinhalten, gezogen (siehe Tabelle 2). Dies resultierte in 100 Print- und 25 Radio- und Fernsehdokumenten.

Tabelle 2: „Kultur“ in Deutschschweizer Mediendokumenten zum Therwil-Fall

\begin{tabular}{|c|c|c|c|c|}
\hline & Printdokumente & $\begin{array}{l}\text { Dokumente mit } \\
\text { Kulturreferenz }\end{array}$ & SRF Outputs & $\begin{array}{r}\text { SRF Outputs mit } \\
\text { Kulturreferenz }\end{array}$ \\
\hline April 2016 & 108 & $57(53 \%)$ & 24 & $16(67 \%)$ \\
\hline Mai 2016 & 19 & $11(58 \%)$ & 7 & $2(29 \%)$ \\
\hline Juni 2016 & 9 & $6(67 \%)$ & 1 & 0 \\
\hline Juli 2016 & 3 & $2(67 \%)$ & 4 & $3(75 \%)$ \\
\hline Aug. 2016 & 5 & $5(100 \%)$ & 0 & 0 \\
\hline Sept. 2016 & 20 & $10(50 \%)$ & 5 & 0 \\
\hline Okt. 2016 & 2 & $2(100 \%)$ & 0 & 0 \\
\hline Nov. 2016 & 1 & 0 & 0 & 0 \\
\hline Dez. 2016 & 8 & $4(50 \%)$ & 7 & $2(29 \%)$ \\
\hline Jan. 2017 & 0 & 0 & 0 & 0 \\
\hline Feb. 2017 & 4 & $1(25 \%)$ & 2 & $2(100 \%)$ \\
\hline März 2017 & 1 & 0 & 6 & 0 \\
\hline April 2017 & 0 & 0 & 0 & 0 \\
\hline
\end{tabular}


Tabelle 2: (fortgeführt)

\begin{tabular}{lrrrr}
\hline & Printdokumente & $\begin{array}{r}\text { Dokumente mit } \\
\text { Kulturreferenz }\end{array}$ & SRF Outputs & $\begin{array}{r}\text { SRF Outputs mit } \\
\text { Kulturreferenz }\end{array}$ \\
\hline Mai 2017 & 4 & 0 & 0 & 0 \\
\hline Juni 2017 & 0 & 0 & 0 & 0 \\
\hline Juli 2017 & 1 & 0 & 2 & 0 \\
\hline Aug. 2017 & 0 & 0 & 0 & 0 \\
\hline Sept. 2017 & 2 & 0 & 0 & 0 \\
\hline Okt. 2017 & 1 & $1(100 \%)$ & 0 & 0 \\
\hline Nov. 2017 & 1 & 0 & 0 & 0 \\
\hline Dez. 2017 & 3 & $1(33 \%)$ & 0 & 0 \\
\hline Total Dokumente & $\mathbf{1 9 2}$ & $\mathbf{1 0 0}$ & $\mathbf{5 8}$ & $\mathbf{2 5}$ \\
\hline
\end{tabular}

In einem ersten Analyseschritt wurden die 125 ausgewählten Dokumente im Rahmen einer qualitativen Inhaltsanalyse auf die explizit mit dem Begriff „Kultur“ verknüpften Inhalte und Deutungen hin analysiert. Dabei wurden drei spezifische Deutungskontexte identifiziert: (1) die Deutung von „Kultur“ als geographisch verortet, (2) die Verknüpfung von „Kultur“ und religiöser Zugehörigkeit und (3) die Auffassung von „Kultur“ als durch Werte konstituiert.

In einem zweiten Schritt wurden die ausgewählten Dokumente im Hinblick auf die Thematisierung dieser drei Deutungskontexte analysiert. Dabei konnten zusätzlich die implizit mit den kulturalisierten Verständnissen von Ort, religiöser Zugehörigkeit und Werten zusammenhängenden Deutungen in die Analyse der Strategien der Kulturalisierung eingebunden werden.

\section{Kulturalisierung im Fall Therwil}

Am Anfang der Berichterstattung wurde der Handschlag der Schweizer „Kultur“ zugeschrieben. Neben der vielzitierten Aussage Simonetta Sommarugas „Der Handschlag ist Teil unserer Kultur ${ }^{\text {"15 }}$ wurden diverse weitere Personen mit ähnlichen Äußerungen rezipiert. So wurde beispielsweise der Präsident des Schweizerischen Lehrerverbandes, Beat Zemp, wiederholt zitiert: „Der Handschlag ist

15 Diese Aussage wurde insgesamt in 34 Zeitungsdokumenten und in 6 weiteren Dokumenten des SRF zitiert. 
Teil unserer Kultur“ (Berner Zeitung Online, 04.04.16; Blick, 05.04.16; Die Südostschweiz, 29.04.16) oder die kantonale Bildungsdirektorin Basellandschaft, Monica Gschwind, stellte klar: „Das Händeschütteln mit Lehrern ist nicht nur ein moralisches Gebot, sondern tief in unserer Gesellschaft und Kultur verwurzelt“ (Basler Zeitung, 20.09.16; Neue Luzerner Zeitung, 21.09.), und „dass man Frauen die Hand schüttelt, das ist in unserer Kultur ein wichtiger Pfeiler“ (Monika Gschwind, SRF, Schweiz Aktuell, 04.04.16). In diesen Attributionen des Handschlages zur Schweizer „Kultur“ zeigt sich eine Deutung der Schweiz als einheitlich handelndes Kollektiv in einem kulturalistischen Idiom.

Über diese Kollektivzuschreibung hinaus wurde der Kulturbegriff mit Deutungskontexten verbunden, die eine kulturalisierende Politisierung ermöglichten. In den folgenden drei Abschnitten wird aufgezeigt, wie in den ersten drei Spielarten der Kulturalisierung die „Eigenarten“ der Schweizer Mehrheitsgesellschaft und der „Muslime“ mit Verweisen auf den Kulturbegriff dargestellt und politisiert wurden. Deutungen von „Kultur“ tauchten in der Medienberichterstattung hinsichtlich geographischer Herkunft (4.1), Religion (4.2) und spezifischer Wertevorstellungen (4.3) auf.

\subsection{Kultur des Ortes}

In der Debatte um die Handschlagverweigerung in Therwil wurde der Kulturbegriff unter anderem verwendet, um Gruppengrenzen mit Verweis auf einen geographischen Ort beziehungsweise auf die geographisch(-ethnische) Herkunft bestimmter Menschen zu definieren. Einerseits wurde dabei von der lokalen „Kultur [hier] in der Schweiz“, oder der „hiesigen Kultur“ gesprochen. ${ }^{16}$ Diese lokal „heimische Kultur“ wurde teilweise über die Schweiz hinaus auf eine größere, „europäische“ oder „westliche“ geographische Verortung hin verstanden. ${ }^{17}$ Andererseits wurde, in Abgrenzung dazu, die syrische Herkunft der zwei Brüder und de-

16 Ausdrücke, die auf dieses ortsgebundene Verständnis von „Kultur“ hinweisen, sind beispielsweise „hiesige Gepflogenheiten“ (Aargauer Zeitung, 06.04.16; Sandra Sollberger, SVP Nationalrätin Basel-Landschaft, Basler Zeitung, 10.06.16), ,hiesiges Kulturgut“ (Christian Amsler, Schaffhauser Erziehungsdirektor, Appenzeller Zeitung, 05.04.16; Wiler Zeitung, 05.04.16), „,unsere“ Hausregeln [hier]“ (Pascal Ryf, CVP-Landrat Basel-Landschaft, Aargauer Zeitung, 01.10.16), „die hiesigen Spielregeln“ (Leserkommentar, Blick, 05.04.16), „,hiesige Werte““ (Geplanter Verfassungsartikel, Bildungsgesetz Basel-Landschaft) oder die „heimische Wertekultur“ (Brigitte Gysin, Co-Präsidentin EVP Basel-Stadt, Basler Zeitung, 11.04.16).

17 Beispiele hiervon sind der Verweis auf ,,westlich geprägte Normen““ (Neue Zuger Zeitung, 15.09.16), „westliche Grundwerte“ (Basler Zeitung, 03.09.16), „westliche Kultur“ (SonntagsBlick, 17.04.16), „Werte [in] Europa“ (Leserkommentar, Blick, 05.04.16) oder auf den Handschlag als 
ren Familie in Therwil hervorgehoben. Dabei wurden die Adjektive „syrisch“ und „muslimisch“ abwechselnd zur Beschreibung der Familie und der Familienmitglieder gebraucht, was ein erster Indikator dafür ist, wie sich im Fokus der übergreifenden Debatte um den Fall Therwil die Kategorien „Religionszugehörigkeit“ und geographisch(-ethnische) Herkunft vermischten.

Die Überschneidung der Kategorien „Religion“ und „Herkunft“ kann in der gleichzeitigen Deutung des Therwil-Falles als ein Problem mit der „Religionsfreiheit“ und ein Problem mit der „Integration“ gesehen werden. Besonders deutlich wird dies beispielsweise in der von der konservativen Schweizer Volkspartei (SVP) eingereichten Interpellation mit dem Titel „Welche Regeln gelten für das gemeinsame Zusammenleben mit Muslimen an den Zuger Stadtschulen? Integration versus Religionsfreiheit“ (Neue Zuger Zeitung, 15.09.16). In diesem Ansatz wird das „Zusammenleben der Kulturen“ zu einem „Zusammenleben mit Muslimen“ und gleichzeitig ein Problem der „Integration“, also mit „Ausländern“. Diese Logik war auch den Handlungen der Regierungsbeamten inhärent: Sie sahen die Handschlagverweigerung als Problem der mangelnden (teilweise sogar fehlenden) Integrationsbereitschaft in die Schweizer Kultur, und als Lösung wurden die Schulen im Kanton Basel gesetzlich verpflichtet, etwaige „Integrationsschwierigkeiten“ dem Amt für Migration zu melden. ${ }^{18}$ Im Zusammenfallen der islambezogenen Problemdeutung (z.B. Religionsfreiheit) und der ausländerbasierten Problemdeutung (z.B. fehlende Integration) wird Religion und (geographische, ethnische) Herkunft im kulturalisiert gedachten muslimischen Subjekt vereint. ${ }^{19}$ Eine ähnliche Ethnisierung von Religion wird auch in einem größeren europäischen Kontext vorgefunden, ${ }^{20}$ so beispielsweise in Deutschland, wo Integrationsprobleme, so Tezcan, heute vermehrt nicht mit Verweis auf „Gastarbei-

„mitteleuropäisches Ritual“ (Der Bund und Der Bund Online, 19.12.16; Tages-Anzeiger und TagesAnzeiger Online, 19.12.16).

18 Konkret spricht Monika Gschwind über die beiden Therwiler Schüler und die Gründe ihrer angestrebten Verfassungsänderung wie folgt: „Ausländerinnen und Ausländer müssen sich integrieren, wenn sie sich in der Schweiz niederlassen wollen.“ (Der Bund, 10.12.16)

19 Diese Überlappung von Religion und Herkunft in der Debatte um den Therwiler Handschlag wurde in der analysierten Berichterstattung einzig durch Diego Stoll, Landrat der sozialdemokratischen Partei (SP), kritisiert: „Es gehe zudem nicht an, dass man Schweizer und ausländische Kinder unterschiedlich behandle, sagt Stoll. Es sei auch nicht durchdacht. ,Was macht man beispielsweise mit islamischgläubigen Kindern, die eingebürgert sind?““(Der Bund Online, 21.02.17)

20 Die gleichzeitige Ethnisierung und Kulturalisierung von religiöser Zugehörigkeit bezeichnet ElTayeb als „new racism“, da „the rise of 'Culture' with a capital C [...] may be most notable in the extent to which it has replaced race in discourses directed at migrants and minorities“ $(2011,83)$. Für weitere Literatur zu „new racism“ siehe Fassin (2012), Modood und Werbner (1997), Stolcke (1995) und Stoler (1995). 
ter“ oder „Ausländer“, sondern mit Verweis auf „die Muslime“ diskutiert werden (2011, 358).

Basierend auf Saïds (1977) einflussreichem Buch Orientalism erläutert Tezcan weiter, dass die „Vorstellung eines geographischen Raumes als eines einheitlichen Kulturraums [als] Medium, in dem sich das europäische Kulturbewusstsein in Absetzung vom Okzident seiner selbst vergewissert“ $(2011,365)$ verstanden werden kann. Diese Vorstellung ist in der Therwiler Mediendebatte ersichtlich, wenn nicht nur der geographische Raum der Schweiz und (mittel-)Europas als einheitlicher Kulturraum aufgegriffen, sondern auch das Bild eines „anderen“, „muslimischen Kulturraumes“ generiert wird. Insbesondere in Leserkommentaren wird das Bild zweier Kulturkreise, ${ }^{21}$ einer ,abendländischen Kultur“ (Blick, 05.04.16; Basler Zeitung, 11.04.16; Thurgauer Zeitung, 07.04.16) und einer „anderen, radikaleren und fundamentaleren“ (Neue Luzerner Zeitung, 14.04.16) und „fremden Kultur“ (Neue Luzerner Zeitung, 14.04.16; Schweiz am Sonntag, 10.04.16; Thurgauer Zeitung, 07.04.16) verwendet. In ähnlicher Art und Weise spricht der als Religions-Experte geladene Hugo Stamm ${ }^{22}$ in einem Interview im SRF davon, dass „diese [islamischen] Gesellschaften [...] prüde [und] sexuell verklemmt“ seien (SRF Kultur Kompakt, 04.04.16). ${ }^{23}$ Die Gegenüberstellung einer „fremden Kultur“, definiert durch die simultane Referenz auf Religion („Muslime“) und einen geographischen Raum²4, führt dabei zur „Schärfung des eigenen Kulturbewusstseins mittels der Profilierung des kulturell Anderen“ (Tezcan 2011,

21 Besonders klar bringt es ein Leserkommentar in der Zeitung Blick wie folgt auf den Punkt: „Die Menschen aus diesem Kulturkreis kommen nach Europa, um an den wirtschaftlichen Errungenschaften teilzuhaben. Sie wollen aber nicht ihre mittelalterlichen Traditionen ablegen, die ja der Grund sind, weshalb es in ihren Ländern nicht gut läuft“ (05.04.16, meine Hervorhebung). Ähnlich wird auch Christian Giordano, Professor für Sozialanthropologie an der Universität Freiburg, in der Weltwoche zitiert: „Giordano begründet seine Forderung damit, man könne Menschen, die aus völlig anderen Kulturkreisen kommen, nicht vollständig in die Schweizer Rechtsordnung integrieren: Die Differenz ist zu gross“ (12.05.16).

22 Hugo Stamm hat Philosophie studiert und ist als Journalist durch seine Recherchen zu religiösen Gruppen wie Scientology bekannt geworden, die er seither unter dem Stichwort „Sekten“ öffentlich thematisiert.

23 Stamms Interview unterstreicht die Ambivalenz der Kultur und Religionsreferenzen in der Debatte, so sagt er einerseits, es habe „nichts mit Religion zu tun, dass ein muslimischer Mann einer Frau nicht die Hand geben darf, im Koran steh[e] nichts davon, [es sei] eine moderne Interpretation [...] [s]eines Erachtens eine Fehlinterpretation“, distanziert hier also Religion und Kultur. Andererseits spricht er aber vom „Kern des Problems“, der Diskrimination der Frau, in ,islamischen Gesellschaften“, wobei er den von ihm problematisierten Kulturraum wieder als ,islamisch“ definiert (SRF Kultur Kompakt, 04.04.16).

24 In dieser Hinsicht wird in Bezug auf den Therwil-Fall oder in Beispielen, die parallel dazu angebracht werden, beispielsweise vom „nahen Osten“ (Jasmin El-Sonbati, Aargauer Zeitung, 
266) und zu einer spezifischen Art des Wissens, mit welchem „der soziale Raum symbolisch aufgespannt und unterteilt wird“ (Tezcan 2011, 358).

Auf einer ersten Ebene der Kulturalisierung führt die Debatte rund um den Therwil-Fall also zur Zuschreibung einer kollektiven Einheit (1) sowohl an die „Kultursubjekte“ im geographischen Raum „Schweiz“ als auch an die in einen anderen geographischen Raum verwiesenen „Muslime“. Gleichzeitig bezieht der Vorwurf der fehlenden Integration die zweite und die dritte Spielart der Kulturalisierung mit ein, so werden im Verweis auf die Schüler, die sich auf Grund ihrer anderen Herkunft nicht an die Schweizer „Kultur“ hielten und sich dahingehend anzupassen hätten, sowohl politisch verantwortliche Subjekte in einem kulturalistischen Idiom konstruiert (2) als auch ein Anspruch auf Kultivierung (3) laut.

\subsection{Kultur und Religion}

Verweise auf Religion und religiöse Zugehörigkeit bilden den zweiten Deutungskontext, mit welchem „Kultur“ in der Medienberichterstattung verbunden wurde. Während in der Talkshow „Arena“ zum Thema „Angst vor dem Islam“ (01.04.16) eine der eingeladenen muslimischen Vertreterinnen, Jasmin El-Sonbati, „Kultur“ als eine von der Religion unabhängige Kategorie verwendete, um das Verhalten der Handschlags-Verweigerung anhand der geographischen Herkunft der Akteure zu verstehen, wird in der darauf folgenden Mediendebatte „Kultur“ vermehrt mit Religion verbunden. So wird beispielsweise die Schweizer „Kultur“ in einigen Artikeln und Leserkommentaren als „christlich geprägt“ (Leserkommentar, Neue Luzerner Zeitung, 14.04.16), „westlich-christliche“ (Leserkommentar, Basler Zeitung, 11.04.16), und als Teil einer „christlichen Tradition“ oder des „christlichen Abendlandes“ (Leserkommentare, Thurgauer Zeitung, 07.04.16) bezeichnet. Hinsichtlich des Vorstoßes der Schweizerischen Christlichen Volkspartei (CVP) gegen „Sonderregelungen zugunsten von Muslimen“ erklärt Pascal Ryf, CVP-Landrat Basel-Landschaft: „,Uns geht es explizit nur um jene Sonderregelungen, die im Widerspruch zu unseren Grundwerten und der gelebten Kultur stehen.' Jüdische Feiertage etwa seien davon nicht betroffen [...] ,Die jüdische Kultur ist Teil der Geschichte der Schweiz““ (Aargauer Zeitung, 06.09.16). ${ }^{25}$ In diesem Sinne scheint

07.04.16; Leserkommentar, Basler Zeitung,) oder von „arabischen Ländern“ (Leserkommentar, Blick, 05.04.16) gesprochen.

25 In einer ähnlichen Weise wird im Weltwoche-Artikel „Pingpong mit orthodoxen Juden“ (14.04.16) eine Unterscheidung zwischen „Juden“ und „Muslimen“ gemacht, um zu erklären, dass die jüdische Bevölkerung „seit hundert Jahren mehr oder weniger stabil“ sei und „niemandem ihren Wertekanon aufdrückt, keinen Einfluss auf unsere Gesellschaft nimmt“, zudem nicht missio- 
es sich bei diesem Vorstoß um eine Grenzziehung des „Eigenen“ durch das Herausarbeiten von „Muslimen“ als das „Andere“ $\mathrm{zu}$ handeln. Dabei wird die „Schweizer Kultur“, wenn auch nicht über ihre Religion definiert, zumindest mit dem Christentum und dem Judentum in Einklang gebracht und von einer „muslimischen Kultur“ abgegrenzt. Die Notwendigkeit politischen Vorgehens gegen „problematische“ religiöse Minderheiten wird damit explizit zum Vorgehen gegen „Muslime“ als die „problematische“ religiöse Minderheit.

Die Kulturalisierung religiöser Gruppen erinnert nicht nur stark an die Kulturkampf-Rhetorik Huntingtons, sondern diese wird in einigen Leserkommentaren auch explizit aufgegriffen, beispielsweise im Leserkommentar, der sagt: „Wir befinden uns [...] in einem Kulturkampf mit dem radikalen Islam“ (Schweiz am Sonntag, 10.04.16), oder im Framing des Bund Online Artikels, in dem der Fall Therwil wie folgt nacherzählt wurde:

„Die Baselbieter Bildungsdirektorin Monica Gschwind, eine Freisinnige, versucht aus der Handschlag-Affäre in Therwil jene staatliche Handlungsanweisung zu basteln, die den anschwellenden Kulturkampf zwischen Christentum und Islam vorzeitig beenden soll.“

(Der Bund Online, 21.02.17, Hervorhebung der Autorin)

In dieser Deutung von Religionen als im Konflikt stehende „Kulturen“ wirkt Kulturalisierung nicht nur in der Spielart der Zuschreibung, sondern es wird gleichzeitig eine Notwendigkeit der Handlung, sowohl im Hinblick auf eine „Verteidigungshaltung“ 26 gegenüber der anderen „Kultur“ als auch in der Formulierung eines „Kultivierungs“- bzw. Anpassungsanspruches gegenüber den „Muslimen“ als „Einwandererbevölkerung“ heraufbeschworen. Dieser „Kultivierungs“-Anspruch zeigte sich in der Forderung, sich an lokale Gepflogenheiten und an ,hiesige Werte“ anzupassen.

niere und ,in keiner Statistik negativ auffällt“. Es müsse daher nicht gegen die „Juden “vorgegangen werden, da deren Lebensstil keine Bedrohung darstelle, „im Gegensatz zur extremistischen Auslegung des Islam, von deren Auswüchsen täglich in den Nachrichten zu hören ist" (Weltwoche, 14.04.16).

26 Formulierungen wie „Wir müssen unsere Werte verteidigen“ (Beat Lüthy, Präsident des Baselbieter Schulleiterverbands, Aargauer Zeitung, 06.04.16) oder „Wir haben in Europa Werte [...] Wir müssen endlich zu unseren Werten und zu unserer Kultur stehen“"(Leserkommentar, Blick, 05.04.16) machen die Notwendigkeit zur Handlung explizit. 


\section{3 „Wertekultur“}

Die Rhetorik eines „Krieges der Kulturen“ bezieht sich auf das Bild eines kulturellen Kollektivs, welches durch spezifische „Werte“ gekennzeichnet sei. Während die meisten Referenzen zu einer „Wertekultur“ in der Mediendebatte um Therwil die Werte unbestimmt ließen, ${ }^{27}$ wurden zwei spezifische Werte implizit oder explizit ausgeführt, nämlich „Freiheit“ und „Geschlechtergleichstellung“. Diese beiden Werte wurden sowohl der „Schweiz“ zugeschrieben als auch als fehlend bei den „Muslimen“ dargestellt.

In einigen Leserkommentaren zeigte sich dabei das Bild der „Muslime“ in der Schweiz als Bedrohung für die „Schweizer Grundwerte und Freiheitsrechte“, also für die Schweiz als „freiheitliche ${ }^{28}$ Gesellschaft“ (Leserkommentar, Basler Zeitung, 09.09.16). Auch SVP-Fraktionschef Dominik Straumann deutete die Handschlagverweigerung hinsichtlich einer Bedrohung der Schweizer „Freiheit“. So wurde er in der Basler Zeitung wie folgt zitiert: „Es geht hier nicht um Bagatellen, sondern darum, bedrohte Freiheitsrechte zu verteidigen“(03.09.16). Andererseits wurde der „muslimischen Kultur“ in emotionaler und höchst negativer Sprache eine fehlende „Gleichstellung der Geschlechter“ zugeschrieben, etwa in der Auslegung des Islam als ein „Kulturgut“, in welchem die „Frauen diskriminiert, diffamiert und verunglimpft [werden]“ (Leserkommentar, Neue Luzerner Zeitung, 14.04.16) und im Verständnis der Handschlagverweigerung als „Frauenverachtung“ (Baselbieter CVP, Basler Zeitung, 05.04.16; Leserkommentare, Basler Zeitung, 11.04.16; 09.09.16; 09.10.17). Ein weiterer Leserkommentar bezeichnete die „Unterdrückung der Frau“ als den „Rassismus des Islam“ und als die „Quintessenz“ des Islam (SonntagsBlick, 17.04.16)29.

27 Unbestimmte Referenzen zu „Schweizer Werten“ sind beispielsweise; „strikt die Werte unserer Gesellschaftsordnung durchsetzen [...] Können wir längerfristig unsere Werte vermitteln?“ (Die Weltwoche, 14.04.16), „die Schweizer Gesellschaft solle ihre Wertekultur erhalten“ (EVP-Basel, Basler Zeitung, 11.04.16), „Heimische ,Wertekultur“ erhalten“ (Basler Zeitung, 11.04.16), „Wer sich nicht an unsere Kulturwerte hält, soll in sein eigentliches Heimatland zurück“ (Leserkommentar, Aargauer Zeitung, 07.04.16), „Schweizer Wertekultur“ (Aargauer Zeitung, 07.04.16), oder „Das steht im krassen Widerspruch zu schweizerischen Werten“ (Neue Luzerner Zeitung, 05.04.16).

28 Beispielsweise in der Bezeichnung der Schweiz als „freiheitlich-demokratisch“ (Leserkommentar, Basler Zeitung, 08.07.16) oder als „freie Gesellschaft“ (Leserkommentar, Basler Zeitung, 06.04.16).

29 In einem anderen Leserkommentar ist von „Verachtung und Diskriminierung“ der Frau die Rede und es wird darauf hingewiesen, dass „davon [...] bei diesen vom Islam geprägten Kindern auszugehen [sei]“ (Leserkommentar, Basler Zeitung, 05.04.16). Eine ähnliche Auslegung ist jedoch nicht nur in Leserkommentaren, sondern auch in der Interpretation von Pascal Ryf, CVP-Landrat, 
Gleichzeitig wird durch die Negation der „Diskriminierung der Frau“ in der Schweiz die Gleichberechtigung von Mann und Frau implizit zu einem Merkmal der Schweiz. ${ }^{30}$ So erklärt beispielsweise der SVP-Politiker Walter Wobmann: „Frauen abwertende Verhaltensweisen haben in der Schweiz keinen Platz“ (Aargauer Zeitung, 27.08.16). ${ }^{31}$ Expliziert wird die Gleichstellung der Frau als Merkmal der Schweiz in einem ausführlichen Leserkommentar zum „Konflikt der Kulturen“ im SonntagsBlick, in welchem von der „Gleichberechtigung der Frau [als der] Quintessenz unserer Freiheit“ und vom „Islam, [als dem] Feind der freien Gesellschaft, zu deren Grundsätzen die Gleichheit der Geschlechter gehört“ (17.04.16) gesprochen wird. In diesem Kommentar werden die beiden für die schweizerische „Wertekultur“ als wesentlich erwähnten Werte zu einem, nämlich der in der Gleichstellung der Geschlechter zum Ausdruck kommenden Freiheit.

Auf dieser Ebene der Kulturalisierung werden einerseits den „Muslimen“ mehr oder weniger homogene Werte zugeschrieben, beziehungsweise ein Fehlen bestimmter Wertevorstellungen im Hinblick auf Geschlechterverhältnisse, um aus „Muslimen“ als „Individuen und Gruppen [für die gouvernementale Rationalität] berechenbare muslimische Subjekte zu konstruieren“ (Tezcan 2011, 358). Dies wird daraus ersichtlich, dass von dem Fall der Handschlagverweigerung in Therwil ausgehend auf ein scheinbar grundsätzliches Problem mit der Diskriminierung von Frauen ${ }^{32}$ geschlossen wurde, welches mit den „Muslimen“ in Zukunft auftreten könnte oder werde, und politische Konsequenzen gefordert werden. ${ }^{33}$

ersichtlich, der sagt, dass „die Frau auf ihre Hülle, auf ihr Geschlecht reduziert ist“ (Aargauer Zeitung, 01.10.16).

30 Die Aussagen zu Geschlechtergleichstellung als fundamentaler Wert der Schweiz scheint in Diskrepanz zur geschichtlichen und rechtlichen Entwicklung der Frauenrechte zu stehen. Genaueres dazu siehe Bürgin und Kühler, diese Zeitschriftenausgabe.

31 Eine ähnliche Zurückweisung kommt von Lilo Lätsch, Präsidentin des kantonalen Lehrerinnen- und Lehrerverbandes, die sagt, dass ,ein Frauenbild dahintersteckt, das in der Schweiz nicht toleriert werden kann“ (Tages-Anzeiger, 05.04.16).

32 Die Auslegung der Handschlagverweigerung als diskriminierend wird lediglich einmal in der Mediendebatte durch Peter Baumann, Basler Religionswissenschaftler, in Frage gestellt, den „die Verknüpfung mit der Diskriminierung der Frau stört: ,Das ist Blödsinn. Es geht nicht um Gleichstellung, denn auch gewisse Musliminnen geben ja Männern die Hand nicht““ (Aargauer Zeitung, 27.05.16).

33 Expliziert wird diese zukünftige Problemlage mit „Muslimen“ in Beiträgen wie „Wir dürfen Diskriminierung von Frauen, Anders- oder Ungläubigen nicht hinnehmen [...] Als Nächstes dürfen keine Mädchen zur Schule, wenn muslimische Knaben diese besuchen.“ (Leserkommentar, Basler Zeitung, 06.04.16), „Was kommt als Nächstes? Verweigerung von Anweisungen einer unverschleierten Lehrerin?“ (Schulleitung Therwil, Basler Zeitung, 07.04.16), oder „Denn solche Probleme [...] wird es in Zukunft eher noch öfter geben “ (Sandra Sollberger, SVP-Nationalrätin Basel-Landschaft, Aargauer Zeitung, 05.09.16). Konkrete Handlungsanleitungen zu den politischen Konsequenzen, 
Neben den ersten zwei Spielarten der Kulturalisierung, (1) der „Zuschreibung“ einer kulturellen Homogenität und der Etablierung einer (2) politischen Strategie, ist im Deutungskontext der „Wertekultur“ auch die dritte Spielart, der (3) Anspruch der Kultivierung, ersichtlich. Dies beinhaltet sowohl eine Selbstbeschreibung der Mehrheitsgesellschaft als auch eine Beschreibung der muslimischen Minderheit, mit denen, basierend auf einem normativen und oft „modernisierungstheoretischen Ablaufnarrati[v] “34, Integrationsansprüche formuliert werden können (Tezcan 2011, 358).

Dieser Anspruch auf Kultivierung der muslimischen Minderheit in der Debatte um Therwil wird deutlich, wenn der Wert der Gleichstellung der Geschlechter zum zentralen Maßstab für mögliche Integration gemacht wird. Eine wichtige Rolle spielte dabei die Baselbieter Bildungsdirektorin Monika Gschwind, die in ihren Stellungsnahmen die Zentralität der Gleichstellung der Geschlechter in der Schweiz und für die Integration in den Vordergrund stellt: „In unserer Gesellschaft gelten gegenüber Frauen und Männern die gleichen Umgangsformen und Verhaltensregeln. Daran will ich konsequent festhalten“ (Aargauer Zeitung, 05.04.16), und „Ausländer müssen sich integrieren, wenn sie sich in der Schweiz niederlassen wollen. Vor allem die Gleichstellung von Männern und Frauen ist für mich unverhandelbar“ (Der Bund; Der Bund Online; Tages-Anzeiger, 19.12.16). Auf eine Nachfrage hinsichtlich des Versuches, die „hiesigen Werte und Rituale“ und die Berücksichtigung „bürgerlicher Pflichten“ in die Basler Verfassung zu schreiben, erklärte die Bildungsdirektorin die Gleichstellung der Geschlechter zum „höchstgehaltenen Wert“ (SRF, Handschlag-Affäre - „Ich habe viel Zuspruch erhalten, nicht nur Kritik.“, 16.12.16). ${ }^{35}$ Auch in weiteren Aussagen wird einerseits auf die „Bringschuld der Ausländer“ hinsichtlich der Integration hingewiesen (Pascal Ryf, Aargauer Zeitung, 06.09.16) und andererseits argumentiert, dass die

die gezogen werden sollen, finden sich beispielsweise bei Philipp Müller, FDP-Ständerat, der sagt „Der Kanton müsse die Betroffenen unverzüglich eine Integrationsvereinbarung unterzeichnen lassen. Darin sei festzuhalten, dass hier ,nur das Schweizer Recht gilt, Schweizer Werte und Gepflogenheiten zu respektieren sind und die Aufenthaltsbewilligung unverzüglich entzogen wird, wenn eine solche Vereinbarung verweigert oder nicht konsequent eingehalten wird““ (Blick, 07.04.16).

34 Auf ein zeitliches Fortschrittsnarrativ deutet beispielsweise der Leserkommentar im Blick, welcher von den „Menschen aus diesem Kulturkreis [und] ihre[n] mittelalterlichen Traditionen [...], die ja der Grund sind, weshalb es in ihren Ländern nicht gut läuft“ (05.04.16, meine Hervorhebung) spricht.

35 Gemäss Monika Gschwind findet die Gesellschaft einen Konsens in Bezug auf Werte und „wir haben einen Wert, der als höchstes gehalten wird, dass Frauen und Männer gleichgestellt sind [...] wenn dies verweigert wird, weil Frauen unrein sind, dann widersetzt sich das diesem Wert [...] bis hierhin und nicht weiter" (SRF, Handschlag-Affäre - „Ich habe viel Zuspruch erhalten, nicht nur Kritik“, 16.12.16). 
Nichtanpassung an die Werte und „gesellschaftlichen Umgangsformen“, wozu gehöre, dass „namentlich keinerlei Unterscheidungen nach dem Geschlecht gemacht“ werde, negativ für die Integration sei, „es vermindere auch ihre Chancen auf dem Arbeitsmarkt“ (Rainer Schweizer, emeritierter Rechtsprofessor, SRF-Artikel, Die Grenzen der Religionsfreiheit, 25.05.16). In dieser Argumentationslinie überwiegt das öffentliche Interesse an der Integration und an der Gleichstellung von Männern und Frauen, oder genauer gesagt, das öffentliche Interesse an der Integration wegen oder im Interesse der Gleichstellung der Geschlechter, die Religionsfreiheit der beiden Schüler in Therwil. In dieser Weise ist in der Debatte um Therwil eine kulturalisierende Politisierung essentialisierter Wertvorstellungen hinsichtlich Geschlechterverhältnissen ersichtlich, die sowohl mit der Vorstellung eines liberalen „Selbst“ als auch mit der Darstellung von „muslimisch ,anderen“ Kultursubjekten“ verbunden werden.

\section{Kritik und Vorwurf der Kulturalisierung}

Die kulturalisierende Auslegung des Handschlages sowie die anschließend geplante Verfassungsänderung blieben nicht unangefochten. Insbesondere das kulturessentialistische Verständnis des Handschlages löste eine Reihe Leserkommentare aus, die den Handschlag als Kulturgut in Frage stellten. ${ }^{36}$ In diesem ersten Kritikpunkt blieb die Prämisse der Auslegung, nämlich dass es um die Abgrenzung dessen geht, was Teil der Schweiz ist und was nicht, intakt. Lediglich das Verständnis des Handschlages als Schweizer Kulturgut wurde hinterfragt. Ein zweiter Kritikpunkt war die geplante Verfassungsänderung, welche die Einhaltung „hiesiger Werte“ und „bürgerlicher Pflichten“ gewährleisten sollte. Dieser Vorstoß wurde als ungenau kritisiert. Hier meldete sich beispielsweise Adil Koller, Präsident der SP Baselland, zu Wort, um auf die Unklarheit der Begriffe „, bürgerliche Pflichten“ und „hiesige Werte“ zu verweisen (Der Bund; Der Bund Online; Tages-Anzeiger 19.12.16). Zudem äußerte sich der SP-Landrat Diego Stoll mit den Worten, es „,bleibt völlig unklar, was mit den ,hiesigen Werten“ auch nur annähernd gemeint sein könnte“ (Der Bund Online, 21.02.17). Auch Pascal Ryf, CVP-Landrat Basellandschaft, der zuvor von „unseren Grundwerten und der gelebten Kultur“ gesprochen

36 Aussagen wie ,der Händedruck als urschweizerisches Kulturgut? Wer genauer hinschaut, wird feststellen: Diese Aussage ist falsch.“(Basler Zeitung, 16.04.16), „Doch seit wann gehört der Händedruck zu unserer Kultur? [...] Ich persönlich habe auch nicht allen meinen Lehrern die Hand gegeben.“ (Die Südostschweiz, 29.04.16), oder „Ich bin Schweizer und würde mich als Christ bezeichnen. Bin aber nicht bereit jedem die Hand zu schütteln.“(Aargauer Zeitung, 10.06.16) kritisieren die Darstellung des Händedruckes als Schweizer „Kulturgut“. 
hatte (Aargauer Zeitung, 06.09.16), äußerte sich nun kritisch gegenüber dem Handschlag-Gesetz, denn es sei „völlig unklar, was mit „hiesigen Werten“ gemeint sei“ (SRF-Artikel, Baselbieter Bürgerliche grundsätzlich für Handschlagpflicht, 27.03.17). Obwohl hierbei kein expliziter Kulturalisierungsvorwurf laut wird, zielt die Kritik darauf ab, ein Verständnis der Schweiz als „Wertekultur“ oder zumindest den Verweis auf solche Werte ohne deren Explikation zu hinterfragen.

Darüber hinaus werden zwei weitere kritische Stimmen in der Debatte laut. Als erstes übt Janine Dahinden, Professorin für transnationale Studien an der Universität Neuchâtel und als Integrationsexpertin in die „Arena“-Sendung „Ein handfestes Problem zwischen Staat und Religion?“ geladen, Kritik an der Kulturalisierung von Geschlechterdifferenz. So sei ,auch die Schweizer Gesellschaft [...] in Teilen noch männlich dominiert - etwa an den Universitäten oder in der wirtschaftlichen Elite. Und würde das Problem nun einzig auf den Islam projiziert, zeige sich ein kurioses Phänomen: ,Plötzlich werden Männer aus dem rechten Spektrum zu Feministen““ (09.04.16). Als zweites äußert Reinhard Schulze, Professor für Islamwissenschaft, als einziger einen mehr oder minder expliziten Kulturalisierungsvorwurf, indem er sagt: „Ich würde erwarten, dass man nicht in diese Art der Rhetorik eines Kulturkampfes eintritt und daraus eine große Sache macht.“ (SRF, Ein Händedruck wird zum Politikum, 05.04.16). Sowohl Janine Dahindens Kritik an der Politisierung kulturalisierter Geschlechterverhältnisse als auch Reinhard Schulzes Vorwurf der kulturalisierenden Kampf-Rhetorik werden jedoch in der fortführenden Printmediendebatte nicht weiter aufgenommen.

\section{Abschließende Bemerkungen}

Die „Handschlag-Affäre“ in Therwil hebt die Fähigkeit der Medien hervor, aus einem Vorkommnis ein mediales Ereignis zu fertigen. So wurde der Vorfall, der sich Monate zuvor ereignete und innerhalb des Schulkontextes bereits beigelegt worden war, zu einem Kristallisationspunkt der medialen Debatte über akzeptable Differenzen im Hinblick auf den Islam in der Schweizer Mehrheitsgesellschaft. In dieser Weise lässt sich der Therwil-Fall als exemplarisch für den zeitgenössischen europäischen und in diesem Fall spezifisch deutschsprachigen Kontext interpretieren. In diesem Kontext werden vermehrt Vorkommnisse, die mit muslimischen Minderheiten in den jeweiligen Gesellschaften im Zusammenhang stehen, zu Ereignissen gemacht, anhand welcher sich Debatten zur Frage nach dem Verhältnis und Zusammenleben von Mehrheitsgesellschaft und muslimischen Minderheiten entfalten (vgl. Langenohl 2011, 84).

Kulturalisierungsspielarten kommen in der medialen Kommunikationsverdichtung um den verweigerten Handschlag in Therwil in vierfacher Weise zum Tra- 
gen; In der Anfangsphase der Mediendebatte werden die Mehrheitsgesellschaft und die Minderheitsgruppe der „Muslime“ anhand der Kategorie „Kultur“ (Spielart 1) als politische Kollektive (Spielart 2) dargestellt und in ein normatives Kultivierungsnarrativ hinsichtlich gesellschaftlicher Integration eingebunden (Spielart 3). Dieses kulturessentialistische Verständnis der Therwiler Handschlagverweigerung wird einerseits von Bundesrätin, Bildungsdirektorium Basellandschaft und von Vertreterinnen und Vertretern des Lehrberufes und der Schulbehörde, andererseits von den rechtskonservativen (SVP), mitte-rechts-wirtschaftsliberalen (FDP) und christdemokratischen (CVP) Politparteien geäußert. Basierend auf dieser Auslegung verwischen die Grenzen der Kategorien „Religion“, „Herkunft“ und „Kultur" in der diskursiven Konstitution eines muslimischen Kollektivs und in der gleichzeitigen Selbstbeschreibung der Schweizer Mehrheitsgesellschaft. Die Kulturalisierung von Geschlechterdifferenz und die damit verbundenen Wertvorstellungen untermauert dabei die drängende Rhetorik der Integrationspolitik.

Kritik an dieser Argumentationskette und an der auf dieser Kulturalisierungslogik basierten Verfassungsänderung wird vereinzelt von Seiten der linken, sozialdemokratischen Partei der Schweiz (SP) und von Wissenschaftsvertreterinnen und -vertretern geäußert. Der Kulturalisierungsvorwurf wird dabei nur einmal expliziert, wenn der übergreifenden Debatte eine Tendenz zur „Kulturkampf“-Argumentation vorgeworfen wird. Im Hinblick auf die Medienberichterstattung und auf den Verlauf der medialen „Handschlag-Affäre“ erschließt sich die Dominanz kulturessentialistischer Auslegungen in der Häufigkeit der plattformübergreifenden Zitation kulturessentialistischer Auslegungen in Mediendokumenten. Auch wenn auf die politische Maßnahme der Verfassungsänderung folgend vermehrt ernüchtert reagiert wurde, überwiegt insgesamt die Kulturalisierungslogik der ersten drei Spielarten in den Kulturreferenzen der analysierten Mediendokumente, was vor allem der Anzahl der Dokumente mit Verweis auf kulturalisierende Auslegungen zu Beginn der Debatte zuzuschreiben ist.

Damit wird in der Debatte um den Therwil-Fall Wissen um Zugehörigkeit zur Schweizer Gesellschaft in der Etablierung einer ,kulturalisierten Mehrheitsgesellschaft“ in Abgrenzung zu einer „muslimischen Minderheit“ mit Referenz zum Kulturbegriff strukturiert. Die „Muslime“ sind in der Logik dieser Auslegung zudem aufgrund ihrer „kulturellen Andersartigkeit“ zu integrieren und sollen sich, um der Integration willen, aktiv „zu den Werten der Aufklärung als Leitkultur einer modernen Gesellschaft verhalten“ (Tezcan 2011, 362). Zentral als solcher Wert hervorgehoben wird dabei die Einstellung zur Geschlechtergleichstellung, was den Fall in einen grösseren europäischen Kontext einreiht, in welchem Sexual- und Geschlechterpolitik in Immigrations- und Integrationsdebatten instrumentalisiert werden (Fassin 2010, 2012). Die scheinbare Durchlässigkeit eines solchen Zugehörigkeitsdiskurses, in welchem lediglich Einstellungen geändert wer- 
den können, um eine erfolgreiche Integration $\mathrm{zu}$ bewerkstelligen, wird durch die gleichzeitige Enthistorisierung und Ethnisierung der „Wertfremdheit“ kulturalisierter muslimischer Subjekte anderer Herkunftsländer erschwert. Die Politisierung von kulturalisierten, geschlechtsspezifischen Wertvorstellungen wirkt dabei als effiziente Abgrenzungsmethode in Immigrations- und Integrationsdebatten.

Im Vergleich zu parallelen Diskursen zu muslimischen Minderheiten in weiteren europäischen Ländern (El-Tayeb 2011, Fassin 2010, 2012, Fassin und Salcedo 2015, Mepschen, Duyvendak und Tonkens 2010) fällt in der Debatte um den Fall Therwil das Ausbleiben zweier Referenzen auf. Einerseits wird in den politisierten Wertvorstellungen lediglich die Geschlechtergleichstellung benannt, diese aber nicht an eine größere Sexualpolitik geknüpft, welche die Toleranz gegenüber Homosexualität als Abgrenzung gegenüber den „Muslimen“ ins Selbstbild der Mehrheitsgesellschaft integriert. Andererseits wird die Schweiz in dieser spezifischen Debatte durch Referenzen zur christlichen und teils jüdischen Religion, sehr selten aber mit Verweis auf einen säkularen Gesellschaftscharakter beschrieben. Die Frage, ob das Weglassen dieser beiden Fortschrittsnarrative, die beispielsweise in Frankreich oder den Niederlanden zentral in der Integrationspolitik auftreten (El-Tayeb 2011, Fassin 2010, 2012, Fassin und Salcedo 2015, Mepschen, Duyvendak und Tonkens 2010), fallspezifisch ist oder auf ein möglicherweise übergreifendes, religionsbezogenes Selbstverständnis beziehungsweise auf eine überwiegend konservative Sexual- und Geschlechterpolitik in der Schweiz hinweist, ist ein interessanter Ansatz für zukünftige, weiterführende Studien.

Funding: Schweizerischer Nationalfonds zur Förderung der Wissenschaftlichen Forschung, Funder Id: http://dx.doi.org/10.13039/501100001711, Grant Number: P0ZHP1_181937

\section{Bibliographie}

Abu-Lughod, Lila. 1996. „Writing against culture.“ In Anthropology in Theory: Issues in Epistemology, hg. v. Henrietta Moore und Todd Sanders, 466-479. Malden: Blackwell Publishing.

Altheide, David L. 1987a. „Ethnographic content analysis.“ Qualitative Sociology 10 (1):65-77. Altheide, David L. 1987b. „Reflections: Ethnographic Content Analysis.“ Qualitative Sociology 10 (1):65-77.

Altheide, David L. und Christopher J. Schneider. 2013. Qualitative Media Analysis. Vol. 38, Qualitative Research Methods. London: Sage.

Asad, Talal. 1997. „Europe against Islam: Islam in Europe.“ The Muslim World 87 (2):183-195.

Behloul, Samuel M. 2011. „Vom öffentlichen Thema zur öffentlichen Religion? Probleme und Perspektiven des Islam im Westen am Beispiel der Schweiz.“ In Religionspolitik - Öffent- 
lichkeit - Wissenschaft: Studien zur Neuformierung von Religion in der Gegenwart, hg. v. Martin Baumann und Frank Neubert, 127-150. Zürich: Pano.

Butler, Judith. 2008. „Sexual politics, torture and secular time.“ British Journal of Sociology 59 (1):59-77.

Dennerlein, Bettina. 2017. „Kulturalisierung transnational. Der Streit um Ehe, Familie und Sexualität im ,Islam ‘. FZG - Freiburger Zeitschrift für GeschlechterStudien 23 (2):37-51.

Dreesen, Philipp, Łukasz Kumięga und Constanze Spieß. 2012. Mediendiskursanalyse: Diskurse - Dispositive - Medien - Macht. Wiesbaden: Springer-Verlag.

El-Tayeb, Fatima. 2011. „Secular Submissions: Muslim Europeans, Female Bodies, and Performative Politics.“ In European Others. Queering Ethnicity in Postnational Europe, 81-120. Minneapolis/London: University of Minnesota Press.

Ezli, Özkan. 2010. „Kultur als Ereignis.“ Fatih Akıns Film ,Auf der anderen Seite“als transkulturelle Narration, Bielefeld.

Fassin, Éric. 2010. „National Identities and Transnational Intimacies: Sexual Democracy and the Politics of Immigration in Europe.“ Public Culture 22 (3):507-529.

Fassin, Éric. 2012. „Sexual Democracy and the New Racialization of Europe.“Journal of Civil Society 8 (3):285-288.

Fassin, Eric und Manuela Salcedo. 2015. „Becoming Gay? Immigration Policies and the Truth of Sexual Identity. “ Archives of Sexual Behavior 44 (5):1117-1125.

Foucault, Michel. 2009. „Diskussion vom 20. Mai 1978.“ In Geometrie des Verfahrens: Schriften zur Methode, hg. v. Daniel Defert und François Ewald, 248-265. Frankfurt am Main: Suhrkamp.

Fraas, Claudia und Michael Klemm. 2005. „Diskurse - Medien - Mediendiskurse. Begriffsklärungen und Ausgangsfragen." In Mediendiskurse. Bestandsaufnahme und Perspektiven, hg. v. Claudia Fraas und Michael Klemm, 1-8. Frankfurt am Main: Peter Lang.

Fraas, Claudia, Stefan Meier und Christian Pentzold. 2014. Online-Diskurse: Theorien und Methoden transmedialer Online-Diskursforschung. Köln: Herbert von Halem Verlag.

Inglehart, Ronald und Pippa Norris. 2003. „The True Clash of Civilizations.“ Foreign Policy 135:63-70.

Kleeberg, Bernhard und Andreas Langenohl. 2011. „Kulturalisierung, Dekulturalisierung.“ Zeitschrift für Kulturphilosophie 5 (2):281-302.

Langenohl, Andreas. 2011. „Öffentliche Reaktionen auf das Schweizer Referendum über Minarettbau und auf ,Deutschland schafft sich ab“.“ Gießener Universitätsblätter 44:83-95.

Madeker, Ellen. 2008. „Deutungsmusteranalyse.“ Türkei und europäische Identität: Eine wissenssoziologische Analyse der Debatte um den EU-Beitritt. Wiesbaden: VS Verlag für Sozialwissenschaften, 115-155.

Mepschen, P., J. W. Duyvendak und E. H. Tonkens. 2010. „Sexual Politics, Orientalism and Multicultural Citizenship in the Netherlands." Sociology. The Journal of the British Sociological Association 44 (5):962-979. doi: 10.1177/0038038510375740.

Modood, Tariq und Pnina Werbner. 1997. The Politics of Multiculturalism in the New Europe: Racism, Identity and Community. London: Palgrave Macmillan.

Okin, Susan Moller. 1999. „Is Multiculturalism Bad for Women?“ In Is Multiculturalism Bad for Women?, hg. v. Joshua Cohen, Matthew Howard und Martha C. Nussbaum, 7-24. Princeton, New Jersey: Princeton University Press.

Pfetsch, Barbara und Frank Marcinkowski. 2009. „Problemlagen der ,Mediendemokratie“ -Theorien und Befunde zur Medialisierung von Politik." In Politik in der Mediendemokratie, 11-33. Springer. 
Reckwitz, Andreas. 2011. „Die Kontingenzperspektive der ,Kultur‘. Kulturbegriffe, Kulturtheorien und das kulturwissenschaftliche Forschungsprogramm." In Handbuch der Kulturwissenschaften, 1-20. Springer.

Sabsay, L. 2012. „The Emergence of the Other Sexual Citizen: Orientalism and the Modernisation of Sexuality." Citizenship Studies 16 (5-6):605-623.

Said, E. W. 1977. „Orientalism.“ Georgia Review 31 (1):162-206.

Schranz, Mario und Kurt Imhof. 2002. „Muslime in der Schweiz - Muslime in der öffentlichen Kommunikation.“ Medienheft 18:1-7.

Stahel, Lea. 2018. „Refusing a Handshake Shakes the World: How Collapsing Contexts Complicate Legitimacy Construction in Networked Publics.“ International Conference on Social Media \& Society, Copenhagen, Denmark.

Stolcke, Verena. 1995. „Talking Culture: New Boundaries, New Rhetorics of Exclusion in Europe.“ Current Anthropology 36 (1):1-24.

Stoler, Ann Laura. 1995. Race and the Education of Desire: Foucault's History of Sexuality and the Colonial Order of Things. Durham/London: Duke University Press.

Tezcan, Levent. 2011. „Spielarten der Kulturalisierung.“ Zeitschrift fur Kulturphilosophie (2):357. Torfing, Jacob. 1999. New Theories of Discourse. Laclau, Mouffe and Zizek. Oxford: Blackwell. Wäckerlig, Oliver. 2014. Das Fanal von Wangen. Der Schweizer Minarettdiskurs - Ursachen und Folgen. Saarbrücken: Akademikerverlag. 